hep-ph/0506206

\title{
Little Higgs Models and Electroweak Measurements
}

\author{
Zhenyu Han* and Witold Skiba ${ }^{\dagger}$ \\ Department of Physics, Yale University, New Haven, CT 06520
}

\begin{abstract}
Using effective field theory approach, we study the constraints from electroweak data on the $S U(5) / S O(5), S U(6) / S P(6), S U(3) \times U(1)$ little Higgs models and their variations. We construct an effective theory valid above the electroweak symmetry breaking scale in these models that includes dimension-six operators induced by integrating out heavy fields. We calculate the constraints on the models using the results of hep-ph/0412166, where bounds on arbitrary linear combinations of flavor and $\mathrm{CP}$ conserving dimension-six operators are given. We present the constraints in terms of the bounds on the masses of heavy fermions and heavy gauge bosons. The constraints are often stringent, but in some regions of the parameter space the constraints are mild enough and do not imply significant fine-tuning.
\end{abstract}

\footnotetext{
* email address: zhenyu.han@yale.edu

$\dagger$ email address: witold.skiba@yale.edu
} 


\section{INTRODUCTION}

Little Higgs models [1-16] have been proposed to stabilize the electroweak scale in the Standard Model (SM), see Ref. [17] for a review. In these models, the one-loop quadraticallydivergent corrections to the Higgs mass from the SM particles are canceled by the corrections from new particles with masses at $\mathrm{TeV}$ scale. Eliminating the one-loop divergences allows the cutoff of the theory to be pushed to about $4 \pi \mathrm{TeV}$, however this could be an optimistic estimate, see Ref. [18]. The predicted particles are likely to be produced and observed in future colliders, especially the LHC. The presence of new particles also creates tension with the electroweak precision tests (EWPTs). To avoid fine-tuning of more than 10\%, heavy fermions and gauge bosons with masses less than about $2 \mathrm{TeV}$ and $5 \mathrm{TeV}$, respectively, should be introduced to cancel the top-loop and gauge-boson-loop divergences. However, the EWPTs do not indicate presence of new particles in a few $\mathrm{TeV}$ range if their couplings are generic. A successful model has to reconcile the tension between naturalness and the EWPTs.

Constraints on little Higgs models from the EWPTs have been considered for different models [19-27]. In this article, we provide a more up-to-date and extensive analysis employing the effective theory approach we described in Ref. [28]. In Ref. [28], we analyzed all flavor-independent and CP-conserving dimension-six operators written in terms of the SM fields that are tightly constrained by EWPTs. We calculated the corrections from these operators to the electroweak precision observables (EWPOs). The result is the $\chi^{2}$ distribution in terms of the coefficients of these operators. In an extension of the SM one can integrate out the heavy fields and obtain the coefficients of the effective operators in terms of parameters in the model. Substituting the coefficients in the $\chi^{2}$ distribution, one immediately obtains global constraints from all EWPOs. As we will discuss, this procedure fits most little Higgs models. We will only consider the tree-level diagrams when we integrate out the heavy fields. Loop diagrams involving heavy fields are usually suppressed by both the masses of the heavy fields and the loop factor. Thus, loop corrections from particles with $\mathrm{TeV}$-scale masses are usually small and do not significantly affect the constraints we obtain. For the discussion of the fine-tuning problem, we will focus on the largest one-loop corrections to the Higgs mass arising from the top quark and the gauge bosons. Alternative estimates of fine-tuning associated with the sensitivity of the Higgs mass to all underlying parameters of little Higgs models are presented in Ref. [29].

In this article, we focus on the following little Higgs models: the $S U(5) / S O(5)$ or the littlest Higgs model [3], the $S U(6) / S P(6)$ [4] model, and the models with the $S U(3) \times U(1)$ gauge group $[6,8,14]$, as well as their variations. For simplicity, from now on we will refer to them as $S U(5), S U(6)$ and $S U(3)$ models respectively, although the first two refer to their global symmetries and the last one refers to its gauge symmetry. The $S U(3)$ little Higgs models can have different global symmetries. In Sec. II, we discuss in general what kind of operators we expect from these models and how to constrain them. Secs. III, IV, V are devoted to detailed discussion of each of the three models. We summarize our results in Sec. VI.

\section{INTEGRATING OUT HEAVY FIELDS}

A complete set of independent dimension-six operators in the SM is given in Ref. [30]. Assuming flavor and CP conservation, in Ref. [28] we narrowed this set down to 21 operators 
that are relevant to EWPTs. In a compact notation, the operators are:

$$
\begin{aligned}
O_{h} & =\left|h^{\dagger} D_{\mu} h\right|^{2}, \\
O_{h f}^{s} & =i\left(h^{\dagger} D^{\mu} h\right)\left(\bar{f} \gamma_{\mu} f\right)+\text { h.c. }, \quad O_{h f}^{t}=i\left(h^{\dagger} \sigma^{a} D^{\mu} h\right)\left(\bar{f} \gamma_{\mu} \sigma^{a} f\right)+\text { h.c. }, \\
O_{f f^{\prime}}^{s} & =\frac{1}{1+\delta_{f f^{\prime}}}\left(\bar{f} \gamma^{\mu} f\right)\left(\overline{f^{\prime}} \gamma_{\mu} f^{\prime}\right), \quad O_{f f^{\prime}}^{t}=\frac{1}{1+\delta_{f f^{\prime}}}\left(\bar{f} \gamma^{\mu} \sigma^{a} f\right)\left(\overline{f^{\prime}} \gamma_{\mu} \sigma^{a} f^{\prime}\right),
\end{aligned}
$$

where $h$ is the SM Higgs doublet, $f, f^{\prime}=q, l, u, d, e$, are the left and right handed fermions. The operators are understood to be summed over flavor indices. The superscripts $s$ and $t$ stand for singlet and triplet $S U(2)$ contractions. For triplet couplings, $f$ has to be a SM doublet $\left(q\right.$ or $l$ ). Note that $O_{h}$ corresponds to the oblique $T$ parameter [31] that breaks the custodial symmetry. We have omitted two operators from our list. The omitted operators are denoted $O_{W B}$ and $O_{W}$ in Ref. [28]. The former corresponds to the oblique $S$ parameter and the latter modifies triple gauge-boson couplings. These two operators are not induced at tree level in the models analyzed here. Four-fermion operators involving only quark fields were not included in our list because they are not constrained as tightly as operators involving some leptons.

Including operators $O_{i}$, we can write the effective Lagrangian as

$$
\mathcal{L}=\mathcal{L}_{S M}+\sum_{i} a_{i} O_{i}
$$

In Ref. [28] we calculated the corrections to EWPOs from the operators $O_{i}$ assuming arbitrary coefficients $a_{i}$. The corrections were combined with known experimental values and the SM predictions to obtain the total $\chi^{2}$ distribution

$$
\chi^{2}=\chi^{2}\left(a_{i}\right)=\chi_{m i n}^{2}+\left(a_{i}-\hat{a}_{i}\right) \mathcal{M}_{i j}\left(a_{j}-\hat{a}_{j}\right),
$$

where $\hat{a}_{i}$ are values of $a_{i}$ that minimize $\chi^{2}$. In Ref. [28] we calculated the corrections to EWPOs to linear order in $a_{i}$, so $\mathcal{M}$ in Eq. (3) is a constant and positive-definite matrix. In a given model, we integrate out the heavy fields and obtain the coefficients $a_{i}$ as functions of the parameters in the model. Expressing $a_{i}$ in Eq. (3) in terms of parameters of a model allows us to immediately obtain constraints on the model without having to compute EWPOs.

Before we list and analyze in detail the coefficients of effective operators in the little Higgs models, we briefly discuss how the effective operators are generated and what interesting features these models have.

There are three kinds of heavy fields in these models: gauge bosons, scalars and fermions. We first discuss the effects of the heavy gauge bosons. The $S U(5)$ and $S U(6)$ models share the same gauge structure: $[S U(2) \times U(1)]^{2}$. The gauge group is broken to the diagonal $S U(2) \times U(1)$ which is identified with the SM gauge group. Thus half of the gauge bosons get masses and the other half remain massless until electroweak symmetry breaking takes place. The heavy gauge bosons include a triplet $W^{\prime}$ and a singlet $Z^{\prime}$, which couple to both the SM Higgs and fermions. The exchange of $W^{\prime}$ generates the triplet coupling operators $O_{h f}^{t}, O_{f f^{\prime}}^{t}$, while the exchange of $Z^{\prime}$ generates $O_{h}, O_{h f}^{s}, O_{f f^{\prime}}^{s}$. The gauge sector in the $S U(3)$ model is quite different: a gauged $S U(3) \times U(1)$ is broken to the $\mathrm{SM} S U(2) \times U(1)$, leaving 5 heavy gauge bosons. One of them behaves like the $Z^{\prime}$ and also induces $O_{h}, O_{h f}^{s}$ and $O_{f f^{\prime}}^{s}$. The others decouple from the light fields. There are no $S U(2)$ triplet operators generated in this case. 
Now we turn to discussing the scalars in the models. In the low-energy spectra, the $S U(5)$ and $S U(3)$ models contain one Higgs doublet and the $S U(6)$ model contains two Higgs doublets. The number of light Higgs doublets is irrelevant in our analysis since only the Higgs vev matters. Besides the doublet, the $S U(5)$ model also contains a heavy triplet scalar. When integrated out, the triplet generates the $O_{h}$ operator. In addition, there are heavy singlets in all the three models, but integrating them out does not generate dimensionsix operators relevant to EWPTs.

Turning to fermions, heavy fermions are needed to cancel the quadratic divergence from the top loop. However, there are often multiple choices that insure the cancelation. In the $S U(5)$ little Higgs model [3], a pair of vector-like heavy fermions is added to the SM fields. The right-handed heavy fermion mixes with the right-handed top quark so that the couplings between the top quark and the SM gauge bosons are modified. The loop divergences from the light two generations do not introduce fine-tuning for a cutoff as low as $4 \pi \mathrm{TeV}$ because the corresponding Yukawa couplings are small. Thus one does not introduce extra fermions to cancel the divergences for the light two generations. Therefore, fermion couplings in this model are flavor-dependent. However, since only the top quark mixes with the heavy fermion and no EWPO involves the top quark in the final or initial states, this flavor-dependent effect is not relevant. Of course, if we added fermions that mix with the first two generations as well, we could generate flavor-dependent operators that do affect EWPTs. Such operators can introduce FCNCs and would be severely constrained. A detailed analysis for this case is beyond the scope of this paper. We will assume approximate flavor-independence in our analysis.

The fermion sector in the $S U(6)$ model is similar to the $S U(5)$ model $^{1}$, while the $S U(3)$ model merits a few more comments. Because the gauge group contains an $S U(3)$, every SM fermion doublet must be combined with an extra fermion to complete an $S U(3)$ triplet. Thus unlike the $S U(5)$ or the $S U(6)$ model, heavy fermions have to be added to all generations. If we assign fermions the same quantum numbers for the three generations and impose the constraint of small FCNCs, we will obtain flavor-independent operators $O_{h q}^{s, t}$ and $O_{h l}^{s, t}$ by integrating out the heavy fermions. This is a result of mixing between the SM and the heavy fermions. This mixing modifies the gauge couplings of the SM fermions.

Besides the operators obtained by integrating out the heavy fields, there exist dimensionsix operators arising from expanding the kinetic term of the nonlinear sigma field to higher orders. It turns out that the dimension-six operators obtained this way do not affect EWPTs in the $S U(5)$ and $S U(3)$ models, while in the $S U(6)$ model, there are contributions to the $O_{h}$ operator when $\tan \beta \neq 1$, where $\tan \beta$ is the ratio of the two Higgs vevs.

\section{THE $S U(5) / S O(5)$ MODEL [3]}

Detailed description of the $S U(5)$ little Higgs model can be found elsewhere. We will only specify the necessary conventions and notation. Throughout this paper, we use $h=\left(h^{+} h^{0}\right)^{T}$ to denote the Higgs doublet, $v$ the vev of the Higgs, and $g$ and $g^{\prime}$ the gauge coupling constants of the SM. The littlest Higgs model is based on a nonlinear sigma model with an $S U(5)$ global symmetry spontaneously broken to its $S O(5)$ subgroup. The $S U(5)$ breaking direction is

\footnotetext{
${ }^{1}$ In Ref. [4], the Yukawa structure also introduces mixing for the bottom quark, but we can make a similar choice as in the SU(5) model. Sec. IV contains an example.
} 
given by the vev of the $\Sigma$ field

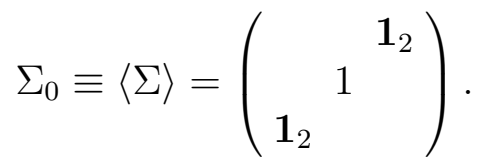

$\Sigma$ can be parameterized around its vev as

$$
\Sigma=e^{2 i \Pi / F} \Sigma_{0}
$$

where the Goldstone boson matrix $\Pi$ is defined as

$$
\Pi=\left(\begin{array}{ccc}
0 & \frac{\tilde{h}}{\sqrt{2}} & \phi^{\dagger} \\
\frac{\tilde{h}^{\dagger}}{\sqrt{2}} & 0 & \frac{\tilde{h}^{T}}{\sqrt{2}} \\
\phi & \frac{\tilde{h}^{*}}{\sqrt{2}} & 0
\end{array}\right) .
$$

In the equation above, $\tilde{h}=i \sigma^{2} h^{*}$, and $\phi$ is a two by two symmetric matrix that represents a scalar triplet with mass $M_{\phi}$ of order $F$. We have omitted the fields that are eaten when the gauge group is broken to the SM gauge group. The kinetic term of the nonlinear sigma model is given by

$$
\frac{F^{2}}{8} \operatorname{Tr}\left[D_{\mu} \Sigma D^{\mu} \Sigma^{\dagger}\right]
$$

where the covariant derivative is defined as

$$
D_{\mu} \Sigma=\partial_{\mu} \Sigma-i \sum_{j=1}^{2}\left[g_{j} W_{j}^{a}\left(Q_{j}^{a} \Sigma+\Sigma Q_{j}^{a T}\right)+g_{j}^{\prime} B_{j}\left(Y_{j} \Sigma+\Sigma Y_{j}^{T}\right)\right] .
$$

$Q_{j}^{a}$ and $Y_{j}$ are the generators of the $[S U(2) \times U(1)]^{2}$ gauge group, defined in the same way as in Ref. [3]. $W_{j}^{a}$ and $B_{j}$ are the corresponding gauge bosons. The mass eigenstates of the gauge bosons are

$$
\begin{aligned}
W & =s W_{1}+c W_{2}, \quad W^{\prime}=-c W_{1}+s W_{2}, \\
B & =s^{\prime} B_{1}+c^{\prime} B_{2}, \quad Z^{\prime}=-c^{\prime} B_{1}+s^{\prime} B_{2},
\end{aligned}
$$

where

$$
s, c=\frac{g_{2}, g_{1}}{\sqrt{g_{1}^{2}+g_{2}^{2}}}, \quad s^{\prime}, c^{\prime}=\frac{g_{2}^{\prime}, g_{1}^{\prime}}{\sqrt{g_{1}^{\prime 2}+g_{2}^{\prime 2}}} .
$$

In Eq. (9), $W$ and $B$ are the SM gauge bosons, and $W^{\prime}$ and $Z^{\prime}$ are heavy gauge bosons with masses

$$
M_{W^{\prime}}=\frac{g F}{2 s c}, \quad M_{Z^{\prime}}=\frac{g^{\prime} F}{\sqrt{20} s^{\prime} c^{\prime}},
$$

where $g=g_{1} s=g_{2} c$ and $g^{\prime}=g_{1}^{\prime} s^{\prime}=g_{2}^{\prime} c^{\prime}$

Most corrections to EWPOs come from the exchanges of the $W^{\prime}$ or $Z^{\prime}$ bosons. Another correction comes from integrating out the triplet field $\phi$ which couples to $h$ as

$$
-i \lambda\left(h^{T} \phi^{\dagger} h-h^{\dagger} \phi h^{*}\right)
$$

where $\lambda$ is a dimensionful coupling of order $F$. The coefficient $\lambda$ is not determined in the lowenergy theory since it arises from quadratically-divergent contributions. When integrated 
out, the triplet generates both dimension-four and dimension-six operators. The dimensionfour operator is the $|h|^{4}$ term contributing to the Higgs potential. In order to insure that the Higgs potential is bounded from below and generates the correct vev for EWSB, the following relation has to be satisfied [21]

$$
\frac{\lambda^{2} F^{2}}{M_{\phi}^{4}}<\frac{1}{4} .
$$

Besides the dimension-four operator, the triplet also generates the dimension-six $O_{h}$ with the coefficient $2 \lambda^{2} / M_{\phi}^{4}$. We will treat $\lambda^{2} / M_{\phi}^{4}$ as a free parameter that is subject to the constraint (13). Note that if we did not integrate out the triplet, after EWSB the triplet would obtain a vev that breaks the custodial symmetry:

$$
v^{\prime}=\langle\phi\rangle=\frac{\lambda v^{2}}{2 M_{\phi}^{2}} .
$$

This is another way to understand the contribution to $O_{h}$.

We assume that fermions are all singlets under the second $S U(2)$ as in Ref. [3], but allow them to be charged under both $U(1)$ 's. The model includes a pair of vector-like heavy fermions to cancel the top loop contribution to the Higgs mass. As explained in Sec. II, the heavy fermions do not affect EWPOs.

Integrating out the heavy fields $W^{\prime}, Z^{\prime}$ and $\phi$, we obtain:

$$
\begin{aligned}
a_{h} & =-\frac{5\left(c^{\prime 2}-s^{\prime 2}\right)^{2}}{2 F^{2}}+\frac{2 \lambda^{2}}{M_{\phi}^{4}}, \\
a_{h q}^{t} & =a_{h l}^{t}=-\frac{\left(c^{2}-s^{2}\right) c^{2}}{2 F^{2}}, \\
a_{h f}^{s} & =\frac{5 s^{\prime} c^{\prime}\left(c^{\prime 2}-s^{\prime 2}\right)}{F^{2}}\left(Y_{2}^{f} \frac{s^{\prime}}{c^{\prime}}-Y_{1}^{f} \frac{c^{\prime}}{s^{\prime}}\right), \\
a_{l q}^{t} & =a_{l l}^{t}=-\frac{c^{4}}{F^{2}}, \\
a_{f f^{\prime}}^{s} & =-\frac{20 s^{\prime 2} c^{\prime 2}}{F^{2}}\left(Y_{2}^{f} \frac{s^{\prime}}{c^{\prime}}-Y_{1}^{f} \frac{c^{\prime}}{s^{\prime}}\right)\left(Y_{2}^{f^{\prime}} \frac{s^{\prime}}{c^{\prime}}-Y_{1}^{f^{\prime}} \frac{c^{\prime}}{s^{\prime}}\right),
\end{aligned}
$$

where $Y_{1}^{f}$ and $Y_{2}^{f}$ are the charges of fermion $f$ under the two $U(1)$ 's. $Y_{1}^{f}$ and $Y_{2}^{f}$ are assumed to be generation independent. The SM hypercharge is $Y=Y_{1}+Y_{2}$. If the $U(1)$ charge assignment is given, we can substitute the coefficients $a_{i}$ into Eq. (3) and obtain $\chi^{2}$ as a function of $f, c, c^{\prime}$ and $\lambda^{2} / M_{\phi}^{4}$.

As we will see shortly, the coefficients $a_{i}$ can put tight constraints on $F$. If $F$ is too large, we will reintroduce fine-tuning to the theory. Therefore it is interesting to consider how to choose the parameters in Eqs. (15) to suppress $a_{i}$. It is easy to see that if $c \ll 1$ and $s^{\prime} \approx c^{\prime}$, the coefficients $a_{h}, a_{h f}^{s}, a_{h f}^{t}$ and $a_{f f^{\prime}}^{t}$ are all suppressed. If we further assume that the fermions are charged equally under the two $U(1)$ 's $\left(Y_{1}^{f}=Y_{2}^{f}\right)$, the coefficients $a_{f f^{\prime}}^{s}$ also vanish. However, it is impossible to render the two $U(1)$ charges equal unless one allows the $U(1)$ 's to be outside of the global $S U(5)$. Changing $Y_{1} \rightarrow Y_{1}+b I$, and $Y_{2} \rightarrow Y_{2}-b I$ 
Z

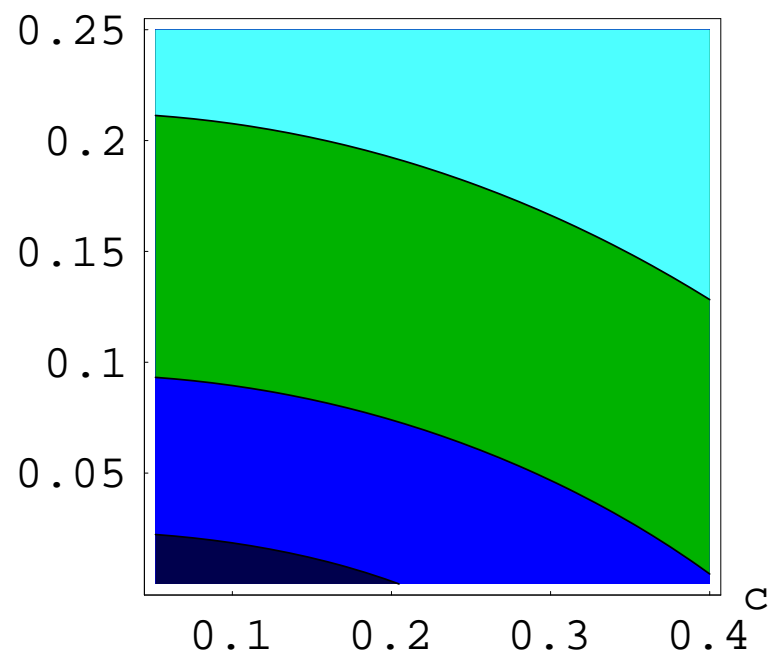

$\mathrm{Z}$

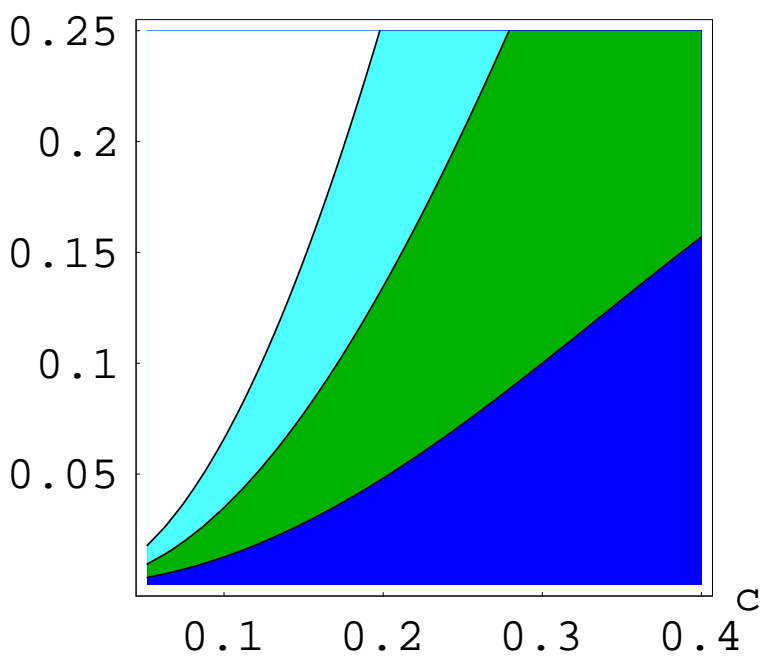

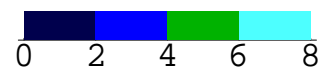

FIG. 1: $95 \%$ CL lower bounds in TeV on $M_{t^{\prime}}$ (left) and $M_{W^{\prime}}$ (right) in the $S U(5)$ model as functions of $c$ and $z \equiv \lambda^{2} F^{2} / M_{\phi}^{4}$ for $Y_{1}^{f}=Y_{2}^{f}$ and $s^{\prime}=c^{\prime}$. In the plots, $c \in[g / 4 \pi, 0.4]$ and $z \in[0,1 / 4]$.

and setting $b=-1 / 80[22]$, we can obtain equal charges ${ }^{2}$. These parameter choices are very much like the "near-oblique" limit for the $S U(6)$ model discussed in Ref. [23]. In this limit, the only significant corrections come from the $\lambda^{2} / M_{\phi}^{4}$ term in $a_{h}$. In addition, $c$ can not be arbitrarily small and has to be greater than $g / 4 \pi$, otherwise the second $S U(2)$ will be strongly coupled.

To make the constraints and thus the associated fine-tuning more transparent, we trade $F$ for the heavy top mass $M_{t^{\prime}}$ and the heavy triplet gauge boson mass $M_{W^{\prime}}$. For the Higgs mass of order $200 \mathrm{GeV}, 10 \%$ fine-tuning corresponds to $M_{t^{\prime}} \sim 2 \mathrm{TeV}$ or $M_{W^{\prime}} \sim 6 \mathrm{TeV}$. $M_{W^{\prime}}$ is given in Eq. (11). As discussed in Ref. [3], $M_{t^{\prime}} \geq \sqrt{2} \lambda_{t} F$, where $\lambda_{t} \sim 1$ is the top Yukawa coupling. For simplicity, we set $M_{t^{\prime}}=\sqrt{2} F$. Fig. 1 shows the bounds on $M_{t^{\prime}}$ and $M_{W^{\prime}}$ around the "near-oblique" limit as functions of $c$ and $\lambda^{2} F^{2} / M_{\phi}^{4}$. We have set $s^{\prime}=c^{\prime}$ and $Y_{1}^{f}=Y_{2}^{f}$ in the two plots. As expected, the bounds on $M_{t^{\prime}}$ are loose near $c=g / 4 \pi$ and $\lambda^{2} f^{2} / M_{\phi}^{4}=0$. On the other hand, because $M_{W^{\prime}} \propto 1 / s c$, the bounds on $M_{W^{\prime}}$ are quite tight near $c=g / 4 \pi$, introducing more fine-tuning than that from the top sector. Nevertheless, there clearly exists a region with less than $10 \%$ fine-tuning. It would be desirable if the charge assignment and the parameter space limit could come naturally from a UV extension

\footnotetext{
${ }^{2}$ In this case, the coefficients listed in Eqs. (15) are modified because of the change in the $Z^{\prime}$ mass. The coefficients $a_{h f}^{t}$ and $a_{f f^{\prime}}^{t}$ do not change while $a_{h f}^{s}, a_{f f^{\prime}}^{s}$ and the term multiplying $\left(c^{\prime 2}-s^{\prime 2}\right)^{2}$ in $a_{h}$ are rescaled by a factor of $1 /\left(1+100 b^{2}\right)$.
} 

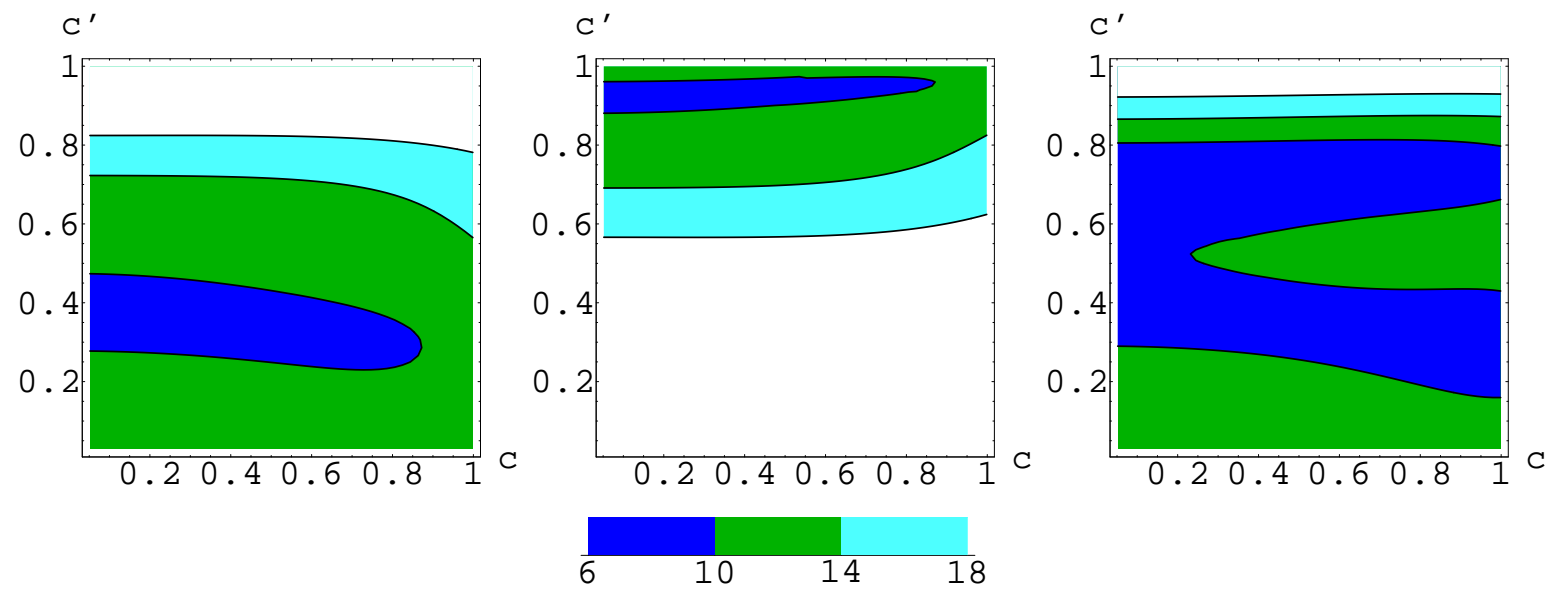

FIG. 2: Lower bounds on $M_{t^{\prime}}$ in TeV in the $S U(5)$ model as a function of $c$ and $c^{\prime}$ at 95\% CL. Left: $U(1)$ charge assignment $Y_{1}^{f}=Y^{f}, Y_{2}^{f}=0$; middle: $Y_{1}^{f}=0, Y_{2}^{f}=Y^{f}$; right: $Y_{1}^{f}=Y^{f}, Y_{2}^{f}=0$ but LEP2 data is excluded. In the plots, $c \in\left[g / 4 \pi, \sqrt{1-(g / 4 \pi)^{2}}\right]$ and $c^{\prime} \in\left[g^{\prime} / 4 \pi, \sqrt{1-\left(g^{\prime} / 4 \pi\right)^{2}}\right]$.

of the model.

Another way to suppress the coefficients in Eqs. (15) is by taking $c, c^{\prime} \ll 1$ and assuming the fermions are charged under only the first $U(1)\left(Y_{1}^{f}=Y^{f}, Y_{2}^{f}=0\right)$. In this limit, all coefficients except $a_{h}$ are suppressed. It turns out that $a_{h}$ alone can still put tight constraints on $F$, as can be seen from Fig. 2. In Fig. 2, we plot the $95 \%$ confidence level (CL) bounds on $M_{t^{\prime}}$ for two fermion charge assignments, as a function of $c, c^{\prime}$. The left (middle) plot corresponds to all fermions charged under only the first (second) $U(1)$. In the plots, we allow both $M_{t^{\prime}}$ and $\lambda^{2} f^{2} / M_{\phi}^{4}$ to vary. The shown bounds on $M_{t^{\prime}}$ are the minimal values for an arbitrary value of $\lambda^{2} f^{2} / M_{\phi}^{4}$ within the [0,1/4] interval. For a two-parameter fit, $\Delta \chi^{2}=5.99$. The limit mentioned above corresponds to the region near the origin in the left plot.

The bounds shown in Fig. 2 are quite stringent. For all of the parameter space, the bounds on $M_{t^{\prime}}$ exceed $6 \mathrm{TeV}$, introducing fine-tuning of more than $1 \%$. This is also true for the parameter limit discussed in the previous paragraph, which makes this limit seem uninteresting. However, as proposed in Ref. [9], it is possible to enlarge one of the $U(1)$ 's to $S U(2)$ and make its coupling relatively strong so that there exists an approximate custodial symmetry that suppresses the coefficient $a_{h}$.

It is worth mentioning that LEP2 data included in our fit contributes significantly to the constraints. For comparison, the plot on the right in Fig. 2 shows the bounds from data excluding LEP2 measurements for the $Y^{f}=Y_{1}^{f}$ case. The bounds are significantly relaxed compared with the bounds obtained using all data. This is because LEP2 experiments are very sensitive to the 4 -fermion operators $O_{f f^{\prime}}^{s, t}$ generated by $Z^{\prime}$ and $W^{\prime}$ exchanges, while LEP1 and other measurements are only sensitive to a few of them.

Other modifications of the model include gauging only $U(1)_{Y}[22]$ and applying a $T$ parity [13]. The former is similar to the first limit discussed above, where the coefficients $a_{h}, a_{h f}^{s}$, $a_{f f^{\prime}}^{s}$ are suppressed due to the lack of the $Z^{\prime}$ boson contribution. The latter avoids generating operators at tree level and thus constraints on the model from EWPTs are less stringent. 
IV. THE $S U(6) / S P(6)$ MODEL [4]

The $S U(6)$ model has the same gauge structure as the $S U(5)$ model but different global symmetry. The nonlinear sigma model terms have the same form as in Eqs. (7) and (8). The $\Sigma_{0}$ and $\Pi$ are six by six matrices:

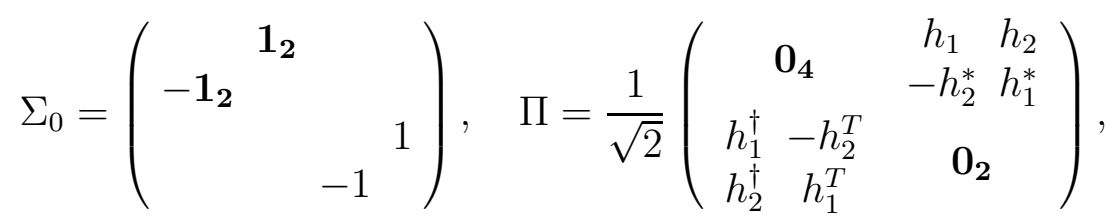

where the two Higgs doublets $h_{1}, h_{2}$ have the $S U(2) \times U(1)$ SM quantum numbers $(\mathbf{2},+1 / 2)$ and $(\mathbf{2},-1 / 2)$, respectively. We have omitted the eaten fields from the $\Pi$ matrix, as well as omitted singlet pseudo-Goldstone bosons that do not affect EWPTs. We have adopted the basis used in Ref. [23] here, but changed the definition of $h_{2}$ for convenience. The $[S U(2) \times U(1)]^{2}$ generators are also given in Ref. [23].

The gauge boson mixings are described by Eqs. (9) and (10) and their masses are

$$
M_{W^{\prime}}=\frac{g F}{2 s c}, \quad M_{Z^{\prime}}=\frac{g^{\prime} F}{\sqrt{8} s^{\prime} c^{\prime}} .
$$

Integrating out the heavy gauge bosons, we obtain

$$
\begin{aligned}
a_{h} & =-\frac{1}{F^{2}}\left[\left(c^{\prime 2}-s^{\prime 2}\right)^{2}+\frac{1}{2} \cos ^{2}(2 \beta)\right], \\
a_{h q}^{t} & =a_{h l}^{t}=-\frac{1}{2 F^{2}}\left(c^{2}-s^{2}\right) c^{2}, \\
a_{h f}^{s} & =\frac{2 s^{\prime} c^{\prime}\left(c^{\prime 2}-s^{2}\right)}{F^{2}}\left(Y_{2}^{f} \frac{s^{\prime}}{c^{\prime}}-Y_{1}^{f} \frac{c^{\prime}}{s^{\prime}}\right), \\
a_{l q}^{t} & =a_{l l}^{t}=-\frac{c^{4}}{F^{2}}, \\
a_{f f^{\prime}}^{s} & =\frac{-8 s^{\prime 2} c^{\prime 2}}{F^{2}}\left(Y_{2}^{f} \frac{s^{\prime}}{c^{\prime}}-Y_{1}^{f} \frac{c^{\prime}}{s^{\prime}}\right)\left(Y_{2}^{f^{\prime}} \frac{s^{\prime}}{c^{\prime}}-Y_{1}^{f^{\prime}} \frac{c^{\prime}}{s^{\prime}}\right) .
\end{aligned}
$$

Since only the vevs of $h_{1}$ and $h_{2}$ matter, we have combined their contributions to a single $h$. The term proportional to $\cos ^{2}(2 \beta)$ in $a_{h}$ comes from expanding the kinetic term in Eq. (7).

The Yukawa couplings can be constructed in several different ways. Here we choose the Yukawas in a way similar to the $S U(5)$ model to avoid extra corrections to EWPOs. For example, we add a pair of fermions $\left(t_{L}^{\prime}, t_{R}^{\prime}\right)$ and define $\chi=\left(u_{3 L}, d_{3 L}, 0,0,0, t_{L}^{\prime}\right)^{T}$. Then the top Yukawa coupling comes from the Lagrangian

$$
\lambda_{1} f \epsilon_{i j k} \epsilon_{x y} \overline{\chi_{i}} \Sigma_{j x} \Sigma_{k y} u_{3 R}+\lambda_{2} f \overline{t^{\prime}}{ }_{L} t_{R}^{\prime},
$$

where $i, j, k \in\{1,2,6\}$ and $x, y \in\{3,4\}$. This Lagrangian only induces mixing between the top quark and the heavy fermion and thus does not affect EWPTs.

Comparing Eq. (15) and Eq. (18), we see that the two sets of coefficients have similar structure. Thus we expect that the constraints from EWPTs in the $S U(6)$ model to have similar features as in the $S U(5)$ model. First, if the fermions are charged under only one 

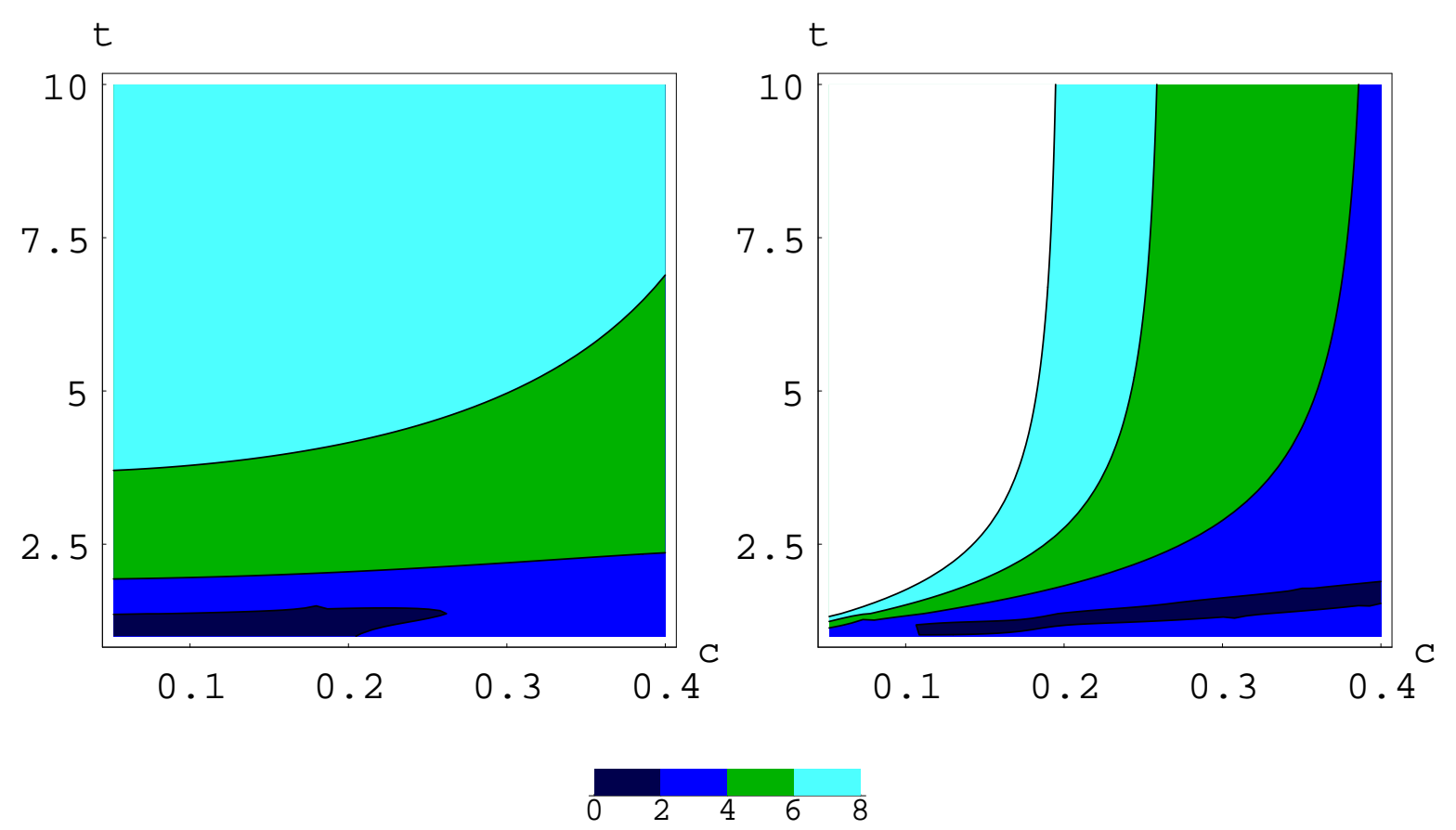

FIG. 3: Lower bounds in TeV on $M_{t^{\prime}}$ (left) and $M_{W^{\prime}}$ (right) in the $S U(6)$ model as functions of $c$ and $t \equiv \tan \beta$ at $95 \% \mathrm{CL}$ for $Y_{1}^{f}=Y_{2}^{f}$ and $s^{\prime}=c^{\prime}$. In the plots, $c \in[g / 4 \pi, 0.4]$ and $t \in[1,10]$.

$U(1)$, the contributions from the $Z^{\prime}$ exchange are large and one expects tight constraints. In this case, we have verified that the bounds on $M_{t^{\prime}}$ are greater than $6 \mathrm{TeV}$ for all choices of $c$, $c^{\prime}$ and $\tan \beta$. Second, the two limits that lead to suppression of coefficients are present here as well. Instead of the triplet contribution in the $S U(5)$ model, there is another custodial symmetry breaking term generated by the nonlinear structure of the $\Sigma$ field in this model. For comparison with the $S U(5)$ model, when $s^{\prime}=c^{\prime}$ and $Y_{1}^{f}=Y_{2}^{f}$ we draw 95\% CL bounds on $M_{t^{\prime}}$ and $M_{W^{\prime}}$ as functions of $c$ and $\tan \beta$ in Fig. 3. The "near-oblique" limit discussed in Ref. [23] corresponds to the region where $\tan \beta \sim 1$ and $c \sim 0$ in Fig. 3. In this limit, the new particles could be so light that loop corrections have to be considered to obtain accurate bounds, as has been done in Ref. [23]. Like in the $S U(5)$ model, the other limit: $Y_{1}^{f}=Y^{f}, Y_{2}^{f}=0$ and $c, c^{\prime} \ll 1$ suppresses all coefficients except $a_{h}$.

The two $U(1)$ generators can also be modified. Since the global symmetry is larger than in the $S U(5)$ model, we have more choices. For example, by redefining

$$
Y_{1} \rightarrow Y_{1}+b I+b^{\prime} \operatorname{Diag}\left\{\mathbf{1}_{2},-\mathbf{1}_{2}, 0,0\right\}, \quad Y_{2} \rightarrow Y_{1}-b I-b^{\prime} \operatorname{Diag}\left\{\mathbf{1}_{2},-\mathbf{1}_{2}, 0,0\right\}
$$

we can rescale the $Z^{\prime}$ mass and the corresponding $a_{i}$. We can also change the $U(1)$ generators in a way that the Higgs bosons are charged differently under the two $U(1)$ 's. For example, taking

$$
Y_{1}=\left(\begin{array}{ccc}
0_{4} & & \\
& -\frac{5}{8} & \\
& & 0
\end{array}\right), \quad Y_{2}=\left(\begin{array}{ccc}
0_{4} & & \\
& \frac{1}{8} & \\
& & \frac{1}{2}
\end{array}\right)
$$


yields the correct $U(1)_{Y}$ charges for $h_{1}$ and $h_{2}$, while the coupling between $h_{2}$ and $Z^{\prime}$ becomes

$$
\frac{1}{2} i g Z^{\prime \mu}\left(D_{\mu} h_{2}\right)^{\dagger}\left(\frac{5 c^{\prime}}{8 s^{\prime}}-\frac{3 s^{\prime}}{8 c^{\prime}}\right) h_{2}+\text { h.c. }
$$

There are similar terms for the $h_{1}$ to $Z^{\prime}$ coupling. Correspondingly, $a_{h}$ and $a_{h f}^{s}$ are no longer proportional to $\left(c^{\prime 2}-s^{\prime 2}\right)$. Such changes affect the near-oblique condition, but they do not introduce essentially new features in our analysis. It would be interesting if such structure comes naturally from some underlying theories.

\section{THE $S U(3) \times U(1)$ MODELS $[\mathbf{6}, \mathbf{8}, \mathbf{1 4}]$}

As effective theories, the little Higgs models do not need to be anomaly free. The original $S U(3)$ model [6] is anomalous, indicating that additional fermions must be present at the cutoff. Anomaly-free versions of the model have also been constructed [14, 15]. However, they require assigning different charges for different generations. Our methods can not be applied to this generation-dependent model. Therefore, we concentrate on the original model only.

By integrating out the heavy fermions, we obtain the following coefficients. The notation follows Ref. [14]. For one generation,

$$
\begin{aligned}
& a_{h l}^{s}=-a_{h l}^{t}=\frac{1}{4} \frac{f_{2}^{2}}{F^{2} f_{1}^{2}}, \\
& a_{h q}^{s}=-a_{h q}^{t}=\frac{1}{4} \frac{\left(\lambda_{1}^{u 2}-\lambda_{2}^{u 2}\right)^{2} f_{1}^{2} f_{2}^{2}}{\left[\left(f_{1} \lambda_{1}^{u}\right)^{2}+\left(f_{2} \lambda_{2}^{u}\right)^{2}\right]^{2} F^{2}},
\end{aligned}
$$

where $F^{2}=f_{1}^{2}+f_{2}^{2}$. For three generations, $\lambda_{1}^{u}$ and $\lambda_{2}^{u}$ are flavor dependent and in general should be $3 \times 3$ matrices. However, in order to avoid large FCNCs, one should set one of the matrices to be proportional to the identity matrix and the other one to be hierarchical [6]. For example, if $\lambda_{2}^{u}$ is proportional to the identity matrix and $\lambda_{1}^{u}$ is hierarchical, for the first two generations, $\lambda_{1}^{u} \ll \lambda_{2}^{u}$, so we can approximate $\lambda_{1}^{u} \simeq 0$. For the third generation, since only the top quark mixes with the heavy quark the EWPTs are not affected. The relation $a_{h q}^{s}=-a_{h q}^{t}$ is a consequence of the fact that the bottom quark does not mix with the heavy fermion. We can assign any values to $a_{h q}^{s}$ and $a_{h q}^{t}$ for the third generation as long as the relation holds. Therefore we can ignore all terms containing $\lambda_{1}^{u}$ in Eq. (24). Similarly, if $\lambda_{2}^{u}$ is hierachical instead, we can ignore all terms containing $\lambda_{2}^{u}$. In this case the coefficients $a_{h q}^{s}$ and $a_{h q}^{t}$ are identical to the coefficients of the corresponding lepton operators in Eq. (23) and the constraints on the model turn out to be even less stringent. ${ }^{3}$ Thus, we obtain the following flavor-independent operators

$$
\begin{aligned}
& a_{h q}^{s}=-a_{h q}^{t}=\frac{1}{4} \frac{f_{1}^{2}}{F^{2} f_{2}^{2}} \quad\left(\lambda_{1}^{u} \text { hierarchical }\right) \\
& a_{h q}^{s}=-a_{h q}^{t}=\frac{1}{4} \frac{f_{2}^{2}}{F^{2} f_{1}^{2}} \quad\left(\lambda_{2}^{u} \text { hierarchical }\right) .
\end{aligned}
$$

\footnotetext{
${ }^{3}$ We thank Heather Logan for drawing our attention to this possibility.
} 
By integrating out the heavy gauge bosons, we obtain

$$
\begin{aligned}
a_{h} & =-\frac{9}{4 F^{2}} \frac{\left(1-\frac{2}{3} x^{2}\right)^{2}}{\left(3+x^{2}\right)^{2}} \\
a_{h f}^{s} & =\frac{9}{4 F^{2}} \frac{1-\frac{2}{3} x^{2}}{\left(3+x^{2}\right)^{2}}\left(\sqrt{3} T^{8 f}+x^{2} Y_{x}^{f}\right), \\
a_{f f^{\prime}}^{s} & =-\frac{9}{2 F^{2}} \frac{1}{\left(3+x^{2}\right)^{2}}\left(\sqrt{3} T^{8 f}+x^{2} Y_{x}^{f}\right)\left(\sqrt{3} T^{8 f^{\prime}}+x^{2} Y_{x}^{f^{\prime}}\right),
\end{aligned}
$$

where $x=g_{x} / g$, while $T^{8 f}=1 /(2 \sqrt{3}), 1 /(2 \sqrt{3}), 0,0,0$ and $Y_{x}^{f}=1 / 3,-1 / 3,2 / 3,-1 / 3,-1$ are fermion charges for $f=q, l, u, d, e$, respectively. Moreover, $g_{x}$ is related to $g$ and $g^{\prime}$ by

$$
g_{x}^{2}=\frac{3 g^{2} g^{\prime 2}}{3 g^{2}-g^{\prime 2}} .
$$

The complete list of coefficients is given by Eqs. (23), (25) and (26).

In this model, the mass of the heavy fermion and the top Yukawa coupling are given by $[14]$

$$
M_{t^{\prime}}^{2}=\lambda_{1}^{2} f_{1}^{2}+\lambda_{2}^{2} f_{2}^{2}, \quad \lambda_{t}=\lambda_{1} \lambda_{2} \frac{F}{M_{t^{\prime}}} .
$$

Unlike the $S U(5)$ and $S U(6)$ models, for given $f_{1}$ and $f_{2}, M_{t^{\prime}}$ is not uniquely determined because of the freedom in choosing the ratios $\lambda_{1} / \lambda_{2}$ and $f_{1} / f_{2}$. Thus, $M_{t^{\prime}}$ is not tightly constrained. However, the mass of the heavy gauge doublet $W^{\prime}$ is determined uniquely in terms of $F: M_{W^{\prime}}^{2}=g^{2} F^{2} / 2$.

Given the ratio $f_{1} / f_{2}$, we can obtain the bound on $M_{W^{\prime}}$. Fig. 4 shows $95 \%$ CL bounds on $M_{W^{\prime}}$ as a function of $f_{1} / f_{2}$. Since the contribution to the Higgs mass from $W^{\prime}$ is not significant until $M_{W^{\prime}}>6 \mathrm{TeV}$ the fine-tuning problem is not severe. Depending on the choice of the Yukawa matrices in the quark sector corresponding to the two possibilities in Eq. (25), the least severe constraints are either when $f_{1} / f_{2} \approx 1$ for hierarchical $\lambda_{1}^{u}$, or when $f_{1} / f_{2}>2$ for hierarchical $\lambda_{2}^{u}$.

The $S U(3) \times U(1)$ gauge sector can be embedded in different global groups. The above discussion makes use of the setup of Ref. [14], where the coset space is $[S U(3) / S U(2)]^{2}$. But the constraints also apply to the model constructed in Ref. [8], where the coset space is $S U(9) / S U(8)$. The model is also extended to an $[S U(4) / S U(3)]^{4}$ model with an $S U(4)$ group gauged in Ref. [6]. These variations mainly stem from theoretical considerations, rather than the need to avoid the experimental constraints.

\section{SUMMARY AND DISCUSSION}

Using effective field theory approach, we have obtained constraints from EWPTs for three types of little Higgs models: $S U(5) / S O(5), S U(6) / S P(6)$ and $S U(3) \times U(1)$. We have carried out the analysis by first integrating out the heavy fields to obtain the effective operators, and then calculating the constraints from the bounds on the linear combinations of the coefficients of these operators.

We gained two main benefits from following through this procedure. First, expressing the corrections in terms of effective operators allowed us to treat the corrections in a modelindependent way, which we have done in Ref. [28]. Thus we can simply use the results in 

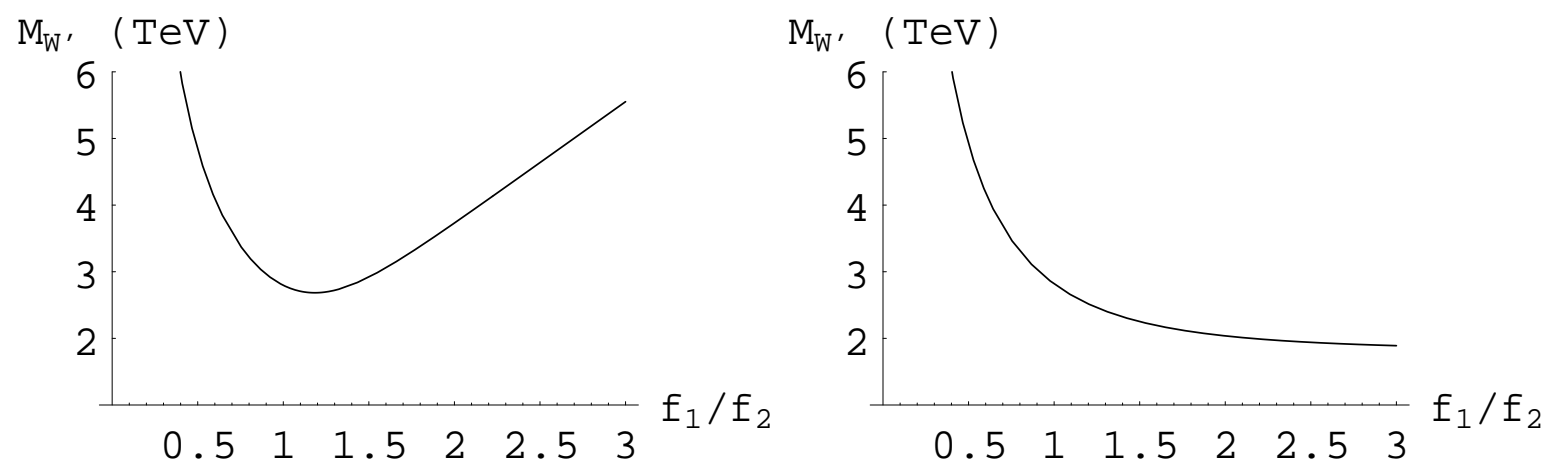

FIG. 4: Lower bounds on $M_{W^{\prime}}$ as a function of $f_{1} / f_{2}$ in the $S U(3)$ models. Left: $\lambda_{1}^{u}$ is hierarchical and $\lambda_{2}^{u}$ is proportional to identity matrix; right: $\lambda_{2}^{u}$ is hierarchical and $\lambda_{1}^{u}$ is proportional to identity matrix.

Ref. [28] once we acquire the coefficients of the operators. For the $S U(5)$ and $S U(6)$ models, we use the heavy top mass $M_{t^{\prime}}$ and the heavy triplet gauge boson mass $M_{W^{\prime}}$ to illustrate the bounds. We show that there exist regions in the parameter space where the bounds on $M_{t^{\prime}}$ and $M_{W^{\prime}}$ are less than $2 \mathrm{TeV}$ and $6 \mathrm{TeV}$ respectively. In these regions, no significant fine-tuning is required to yield light Higgs bosons. These regions are in the vicinity of the points where the two $U(1)$ gauge sectors in the models have the same coupling strength and fermions are charged identically under the two $U(1)$ 's. Away from this limit, the bounds are usually much tighter. In particular, for the cases that fermions are charged under only one $U(1)$, we obtain the bounds $M_{t^{\prime}}>6 \mathrm{TeV}$ at 95\% CL for all of the parameter space, requiring fine-tuning of more than $1 \%$. For the $S U(3)$ model, $M_{t^{\prime}}$ remains a free parameter for a given $F$. Thus we only obtain the bounds for $M_{W^{\prime}}$. At $95 \% \mathrm{CL}, M_{W^{\prime}}>1.8 \mathrm{TeV}$. The associated fine-tuning is not significant in this case.

It is interesting that LEP2 results are very useful. The models induce many 4-fermion operators which LEP2 observables are sensitive to. The LEP2 results were not included in most of previous papers considering EWPT constraints on littlest Higgs models. As a comparison, we have checked our results for the $S U(5)$ and $S U(6)$ models against Ref. [19, 22]. We agree with the results in the two references when we use the same set of observables, which contain most precisely measured LEP1 and some low-energy observables. After we include the LEP2 observables, the bounds become significantly tighter.

In Ref. [27] the authors also obtain constraints on little Higgs models from EWPTs including LEP2 data. Technically, our approach is more general. The authors of Ref. [27] have to assume that the models are approximately universal and all corrections can be condensed in four oblique parameters. In particular, the heavy gauge bosons should couple to fermions "universally", which means that the fermion currents that couple to the heavy gauge bosons are proportional to the SM current. This is not true for the $S U(3)$ model, so the authors of Ref. [27] have to neglect some of the EWPOs. In addition, they do not discuss the effects of the heavy fermions in the $S U(3)$ model, which are not universal either. In the $S U(5)$ and $S U(6)$ models we have analyzed, the fermion charge assignments do yield universal couplings between fermions and gauge bosons. Thus their method apply as well. 
However, one could obtain non-universal couplings from more general fermion assignments, which would make their method inapplicable, but would not introduce any difficulty in our approach. In [27] only the simplest fermion assignments made in the original papers were considered, while we are interested in the variations which relax the electroweak constraints.

The second advantage of the effective theory approach is that it makes it transparent how to modify the models to avoid tight constraints. From the compact but complete lists of operator coefficients in the $S U(5)$ and $S U(6)$ models, we easily deduce two regions of parameter space that lead to suppressions of the corrections. One of the regions for the $S U(6)$ model case is discussed and termed "near-oblique" limit in Ref. [23]. In Ref. [9], the other limit has been utilized in a model with enlarged gauge sector and an approximate custodial symmetry. With their weaker constraints, such model variations are interesting since they address the fine-tuning problem better than the original models. From experimental point of view, new particles are light enough to be observable at the LHC only if there is no significant fine tuning.

\section{Acknowledgments}

This research was supported in part by the US Department of Energy under grant DEFG02-92ER-40704 and by Outstanding Junior Investigator grant. WS thanks the Aspen Center for Physics for its hospitality.

[1] N. Arkani-Hamed, A. G. Cohen and H. Georgi, Phys. Lett. B 513, 232 (2001) [arXiv:hepph/0105239].

[2] N. Arkani-Hamed, A. G. Cohen, E. Katz, A. E. Nelson, T. Gregoire and J. G. Wacker, JHEP 0208, 021 (2002) [arXiv:hep-ph/0206020].

[3] N. Arkani-Hamed, A. G. Cohen, E. Katz and A. E. Nelson, JHEP 0207, 034 (2002) [arXiv:hep$\mathrm{ph} / 0206021]$.

[4] I. Low, W. Skiba and D. Smith, Phys. Rev. D 66, 072001 (2002) [arXiv:hep-ph/0207243].

[5] M. Schmaltz, Nucl. Phys. Proc. Suppl. 117, 40 (2003) [arXiv:hep-ph/0210415].

[6] D. E. Kaplan and M. Schmaltz, JHEP 0310, 039 (2003) [arXiv:hep-ph/0302049].

[7] S. Chang and J. G. Wacker, Phys. Rev. D 69, 035002 (2004) [arXiv:hep-ph/0303001].

[8] W. Skiba and J. Terning, Phys. Rev. D 68, 075001 (2003) [arXiv:hep-ph/0305302].

[9] S. Chang, JHEP 0312, 057 (2003) [arXiv:hep-ph/0306034].

[10] H. C. Cheng and I. Low, JHEP 0309, 051 (2003) [arXiv:hep-ph/0308199].

[11] H. C. Cheng and I. Low, JHEP 0408, 061 (2004) [arXiv:hep-ph/0405243].

[12] D. E. Kaplan, M. Schmaltz and W. Skiba, Phys. Rev. D 70, 075009 (2004) [arXiv:hep$\mathrm{ph} / 0405257]$.

[13] I. Low, JHEP 0410, 067 (2004) [arXiv:hep-ph/0409025].

[14] M. Schmaltz, JHEP 0408, 056 (2004) [arXiv:hep-ph/0407143].

[15] O. C. W. Kong, Phys. Rev. D 70, 075021 (2004) [arXiv:hep-ph/0409238].

[16] J. Thaler and I. Yavin, arXiv:hep-ph/0501036.

[17] M. Schmaltz and D. Tucker-Smith, arXiv:hep-ph/0502182.

[18] S. Chang and H. J. He, Phys. Lett. B 586, 95 (2004) [arXiv:hep-ph/0311177]. 
[19] C. Csaki, J. Hubisz, G. D. Kribs, P. Meade and J. Terning, Phys. Rev. D 67, 115002 (2003) [arXiv:hep-ph/0211124].

[20] J. L. Hewett, F. J. Petriello and T. G. Rizzo, JHEP 0310, 062 (2003) [arXiv:hep-ph/0211218].

[21] T. Han, H. E. Logan, B. McElrath and L. T. Wang, Phys. Rev. D 67, 095004 (2003) [arXiv:hep$\mathrm{ph} / 0301040]$.

[22] C. Csaki, J. Hubisz, G. D. Kribs, P. Meade and J. Terning, Phys. Rev. D 68 (2003) 035009 [arXiv:hep-ph/0303236].

[23] T. Gregoire, D. R. Smith and J. G. Wacker, Phys. Rev. D 69, 115008 (2004) [arXiv:hep$\mathrm{ph} / 0305275]$.

[24] M. C. Chen and S. Dawson, Phys. Rev. D 70, 015003 (2004) [arXiv:hep-ph/0311032].

[25] R. Casalbuoni, A. Deandrea and M. Oertel, JHEP 0402, 032 (2004) [arXiv:hep-ph/0311038].

[26] W. Kilian and J. Reuter, Phys. Rev. D 70, 015004 (2004) [arXiv:hep-ph/0311095].

[27] G. Marandella, C. Schappacher and A. Strumia, arXiv:hep-ph/0502096.

[28] Z. Han and W. Skiba, Phys. Rev. D 71, 075009 (2005) [arXiv:hep-ph/0412166].

[29] J. A. Casas, J. R. Espinosa and I. Hidalgo, JHEP 0503, 038 (2005) [arXiv:hep-ph/0502066].

[30] W. Buchmuller and D. Wyler, Nucl. Phys. B 268, 621 (1986).

[31] M. E. Peskin and T. Takeuchi, Phys. Rev. D 46, 381 (1992). 